\title{
Hysterosalpingogram in evaluation of primary and secondary infertility: a two year study at a tertiary care hospital of South India
}

\section{Gurram Swetha Reddy, Gandavaram Jyothi*}

Department of Obstetrics and Gynecology, Narayana Medical College, Andhra Pradesh, India

Received: 02 May 2019

Accepted: 16 May 2019

\section{*Correspondence:}

Dr. Gandavaram Jyothi,

E-mail: sujatha2481@gmail.com

Copyright: (C) the author(s), publisher and licensee Medip Academy. This is an open-access article distributed under the terms of the Creative Commons Attribution Non-Commercial License, which permits unrestricted non-commercial use, distribution, and reproduction in any medium, provided the original work is properly cited.

\begin{abstract}
Background: Infertility globally is a raising threat and problem associated with significant social and psychological problem. Infertility currently affects about 10 to 14 percent of the Indian population, with higher rates in urban areas where one out of six couples is impacted. Patency of the tubes, structure of uterus and cervix can be visualized by hysterosalpingogram which is a simple safer inexpensive investigation of choice in cases of infertility. Objective of this study was to study the role of hysterosalpingography (HSG) in cases of primary and secondary infertility and to observe the structural abnormalities of cervix, uterus and fallopian tubes.

Methods: A two year prospective study was conducted on cases of primary and secondary infertility attending the OPD of infertility clinic. HSG was performed on the subjects included in study and findings were noted. Analysis was done by Microsoft excel spread sheet.

Results: A total of 286 cases with $11.19 \%$ of primary infertility and $88.81 \%$ of secondary infertility evaluated by HSG. Mean age of the study group was $35.16 \pm 2.8$ years. $74.83 \%$ had abnormal findings, with $20.28 \%$ having normal uterine cavity with uterine filling defect being the common abnormality. Two congenital anomalies of uterus were noted. Tubal occlusion was seen in $8.39 \%$ right and $9.09 \%$ left cases. Hydrosalpinx was observed in $7.69 \%$ of right and $9.79 \%$ of left cases.

Conclusions: To conclude, women presenting with infertility should be evaluated for tubal pathology. The tubal pathologies like occlusion or hydrosalpinx may be due to infections or post surgical sequelae. HSG remains one of the diagnostic backbone in infertility even availability of new modalities.
\end{abstract}

Keywords: Hysterosalpingogram, Hydrosalpinx, Infertility, Primary infertility, Secondary infertility

\section{INTRODUCTION}

Infertility globally is a raising threat and problem associated with significant social and psychological problem. Global statistics reveal that about 50 million couples worldwide experience infertility with various reasons. The clinical definition of infertility provided by the World Health Organization is "a disease of the reproductive system defined by the failure to achieve a clinical pregnancy after 12 months or more of regular unprotected sexual intercourse." A number of factors including genetics, environmental exposures and infectious diseases have been linked to infertility risk. ${ }^{1}$ Infertility is defined as primary if the couple fails to conceive even after 12 months of unprotected sexual intercourse and secondary if inability to get pregnant after an earlier pregnancy which may or may not have led to live birth. ${ }^{2}$ According to the Indian Society of Assisted Reproduction, infertility currently affects about 10 to 14 percent of the Indian population, with higher rates in urban areas where one out of six couples is impacted. Nearly 27.5 million couples actively trying to conceive 
suffer from infertility in India. Sexually transmitted infections, polycystic ovarian syndrome, fibroids, and genital TB are other new areas of concern among women. A rise in unprotected sex had led to sexually transmitted infections (STIs) and widespread use of both emergency contraception and surgical abortions, which can trigger serious infections that may cause irreversible infertility. Among the various external factors contributing for infertility Rapid urbanization, job pressures, vehicular pollution, and postponing parenthood are quoted in various studies as other salient reasons for infertility in India. $^{3}$

Multiple factors are associated with causation of infertility in both and women. In females, this could be caused by disorders of cervix, uterus, fallopian tubes and ovaries. But $35-40 \%$ of causes are due to disorders in fallopian tubes. A patent and functional fallopian tube is a prerequisite for fertility, these play a critical role in fertility and also they are easily vulnerable to infections and surgical damage. Any damage to the tube impairs the function by affecting the delicate fimbriae or highly specialized endosalpinx. Patency of the tubes, structure of uterus and cervix can be visualized by hysterosalpingogram which is a simple safer inexpensive investigation of choice in cases of infertility. Some of the studies have suggested HSG as some therapeutic role in enhancing subfertility. ${ }^{4}$

The present study was aimed to study the role of hysterosalpingography (HSG) in cases of primary and secondary infertility and to observe the structural abnormalities of cervix, uterus and fallopian tubes.

\section{METHODS}

The present study was conducted by department of Obstetrics and Gynaecology in association with Department of Radio diagnosis. The study was conducted for a period of two years from January 2016 to December 2017.The study population included all the cases aged between 20-40 years attending the infertility clinic organized by Department of obstetrics. These cases were screened initially for all the causes of infertility by a thorough clinical history and physical examination. Subjects fulfilling the inclusion criteria of the study were enrolled in the study.

The study was presented before the institutional ethical committee and consent was approved. The study was conducted as per the guidelines of the committee. The subjects included in the study were clearly explained about the study procedure and details and a written informed consent was obtained from the subjects. The socio demographic data of the study subjects, including a marital history of marriage, previous history of miscarriages, menstrual history, and contraceptive usages were obtained by interviewing and noted in a separate predesigned questionnaire sheet.

\section{Investigations}

General investigations for the study subjects were haemoglobin estimation, VDRL, urine routine microscopy and semen analysis of the partner to rule out male cause of infertility.

\section{Inclusion criteria}

- Married women between the age group of 20-40 years with primary /secondary infertility willing for infertility workup

- Confirmed ovulatory cycles or ovarian reserve

- Absence of severe sperm pathology in male partner.

\section{Exclusion criteria}

- Presence patients who didn't turn up after HSG

- Past history of pelvic surgical procedures

- Women who became pregnant after HSG

- $\quad$ Subjects allergic to contrast material.

\section{HSG technique}

HSG was performed by a trained radiologist. Verbal informed consent was obtained from all the cases after explaining the procedure and reassurance. The procedure was performed between 6-12 days of the menstrual period because the endometrium will be thin during proliferative phase and also facilitates image interpretation and ensures no existing pregnancy. Patient was placed on fluoroscopy table and scout film of the pelvis was acquired to assess the technical factors and radiopaque pelvic lesions. $15-20 \mathrm{ml}$ contrast medium (Urograffin $76 \%$ ) was injected into uterine cavity through a canula placed in the cervical canal. The appearance of the uterine cavity and patency of the fallopian tubes were assessed by image intensification. Spot films were taken initially to early uterine filling; tubal filling and peritoneal spill were taken.

All HSG interpretations were done by direct visualization of the hard copy images, checking for bilateral or unilateral spillage of contrast medium and abnormalities in the outline of the cervix and uterine cavity. HSG is a outpatient radiological procedure under fluoroscopic guidance by a radiologist taken at different intervals during the examination.

\section{Normal}

Well tubal outlining by free flow of the dye, without loculation in the peritoneal cavity.

\begin{abstract}
Abnormal
Evidence of either unilateral or bilateral tubal obstruction or peritubal adhesions.
\end{abstract}




\section{Statistical analysis}

All the data was entered in a Microsoft excel spread sheet and corrected. The categorical data was expressed as rates, ratios and proportions and continuous data was expressed as Mean \pm standard deviation.

\section{RESULTS}

In the present study conducted for a period of two years a total of 4817 cases attended the infertility clinic and 286 cases were included as subjects who fulfilled the inclusion criteria of the study. There were 32 cases of Primary Infertility $(11.19 \%)$ and 254 cases $(88.81 \%)$ of secondary infertility. In Toto, majority of cases were in the age group of 36-40 years and the mean age of the study group was $35.16 \pm 2.8$ years. Mean age of cases of primary infertility was $27.28 \pm 3.8$ years and in cases of secondary infertility the mean age of the cases was
$27.21 \pm 6.5$ years. In the study, $82 \%$ were literate and above secondary grade with a brief knowledge on infertility and $18 \%$ were illiterate with no idea about infertility. Based on the modified Kuppuswamy classification of socio economic status majority of the cases were in lower middle class with 57\%, 28\% upper lower and $15 \%$ upper middle class. $73 \%$ of cases of primary infertility were of lower middle class and $68 \%$ of secondary infertility cases. History of consanguineous marriage was observed in only $12 \%$ of cases in the study and was found not significant in our study. In our study, $88.81 \%$ of cases of infertility had regular cycles and $11.19 \%$ complained of irregular cycles. Regular cycles were reported in $75 \%$ of cases of primary infertility and $90.6 \%$ cases of secondary infertility. Mean duration of primary and secondary infertility was $5.42 \pm 2.6$ years and $5.34 \pm 3.5$ years respectively. Maximum number of cases had duration of infertility between 1-4 years (54\%) (Table 1).

Table 1: Socio demographic profile of cases in the study.

\begin{tabular}{|llll|}
\hline Age $($ Years) & Primary & Secondary & Total $(\%)$ \\
\hline $20-25$ & 12 & 41 & $53(18.53)$ \\
\hline $26-30$ & 8 & 72 & $80(27.97)$ \\
\hline $31-35$ & 8 & 61 & $69(24.13)$ \\
\hline $36-40$ & 4 & 80 & $84(29.37)$ \\
\hline Total & 32 & 254 & $286(100)$ \\
\hline Menstrual cycles & & & $254(88.81)$ \\
\hline Regular & 24 & 230 & $32(11.19)$ \\
\hline Irregular & 8 & 24 & $229(80.07)$ \\
\hline Literacy & & & $57(19.93 \%)$ \\
\hline Literate & 6 & 223 & \\
\hline Illiterate & 26 & 31 &
\end{tabular}

Table 2: Clinical profile and findings in cases of the study.

\begin{tabular}{|llll|}
\hline Version of uterus & Primary & Secondary & Total (\%) \\
\hline Anteverted & 28 & 244 & $272(95.10)$ \\
\hline Retroverted & 4 & 10 & $14(4.90)$ \\
\hline Size of uterus & & & $248(86.71)$ \\
\hline Normal & 28 & 220 & $38(13.29)$ \\
\hline Bulky & 4 & 34 & $232(81.12)$ \\
\hline Menstrual history & & & $13(4.55)$ \\
\hline Normal & 29 & 203 & $23(8.04)$ \\
\hline Oligomenorrhoea & 1 & 12 & \\
\hline Menorrhagia & 2 & 21 & \\
\hline
\end{tabular}

On clinical examination, bimanual examination of the uterus revealed that $95.01 \%$ were anteverted and $4.9 \%$ were retroverted. Majority were anteverted in both primary $(87.5 \%)$ and secondary infertility $(96.06 \%)$. In the present study, bulky uterus was noted in $13.29 \%$ of cases and $86.71 \%$ had normal sized uterus. $81.12 \%$ cases reported normal menstrual history with $4.55 \%$ cases oligomenorrhoea and $8.04 \%$ menorrhagia (Table 2).

\section{HSG findings}

In the present study, seventy two cases $(25.17 \%)$ had no pathology whereas 214 cases (74.83\%) had pathologies in the uterus, cervix, and fallopian tubes or in more than one of the structures. Majority of the cases with abnormal findings were reported in secondary infertility $(81.89 \%)$. 
Table 3: Pattern of uterine findings among cases of study on HSG.

\begin{tabular}{|llll|}
\hline & Primary & Secondary & Total (\%) \\
\hline Normal findings & $26(81.25 \%)$ & $46(18.11 \%)$ & $72(25.17 \%)$ \\
\hline Abnormal findings & $6(18.75 \%)$ & $208(81.89 \%)$ & $214(74.83 \%)$ \\
\hline Total & $\mathbf{3 2}$ & $\mathbf{2 5 4}$ & $\mathbf{2 8 6}$ \\
\hline Uterine findings on HSG & & & \\
\hline Normal uterine cavity & $20(34.48 \%)$ & $38(65.52 \%)$ & $58(20.28 \%)$ \\
\hline Smooth outline & $10(4.72 \%)$ & $202(95.28 \%)$ & $212(74.13 \%)$ \\
\hline Irregular contour & 0 & $14(100 \%)$ & $14(4.9 \%)$ \\
\hline congenital anomaly & $2(100 \%)$ & 0 & $2(0.7 \%)$ \\
\hline
\end{tabular}

Table 4: Pattern of fallopian abnormalities on HSG.

\begin{tabular}{|lll|}
\hline Fallopian tube pattern & Right Tube (\%) & Lefit tube (\%) \\
\hline Tube not demonstrated & $42(14.69)$ & $44(15.38)$ \\
\hline Fully demonstrated tubes & $148(51.75)$ & $152(53.15)$ \\
\hline Free contrast spill & 138 & 145 \\
\hline Loculated contrast spill & 10 & 7 \\
\hline Tube occlusion & $24(8.39)$ & $26(9.09)$ \\
\hline Cornual occlusion & 2 & 3 \\
\hline Isthmic obstruction & 2 & 3 \\
\hline Distal obstruction & 20 & 20 \\
\hline Hydrosalpinx & $22(7.69)$ & $28(9.79)$ \\
\hline No contrast spill & 18 & 22 \\
\hline Free contrast spill & 2 & 2 \\
\hline Loculated contrast spill & 2 & 4 \\
\hline
\end{tabular}

\section{Uterine findings}

Normal uterine cavity was found in 58 cases $(20.28 \%)$ with $34.48 \%$ among primary infertility and $65.52 \%$ in cases of secondary infertility. Smooth uterine lining was observed in 212 cases $(74.13 \%)$ and irregular contour in $4.9 \%$ of cases. All cases with irregular contour were observed in cases of secondary infertility. Two congenital anomalies of the uterus $(0.70 \%)$, one case of unicornuate and one case of bicornuate uterus was observed in cases of primary infertility. Bulky uterus on HSG was noted in $14.69 \%$ of cases, normal in $81.82 \%$ and small sized in $3.5 \%$ of cases. Of the 42 cases with bulky uterus 27 cases (64.29\%) had leiomyoma and 6 cases with leiomyoma had normal sized uterus. On HSG, four cases of cervical abnormalities were observed one with synechiae and three with irregularity (Table 3).

\section{Tubal findings}

Table 4 summarizes the tubal findings on HSG. 42 right tubes $(14.69 \%)$ and 44 left tubes $(15.38 \%)$ were not visualized completely, whereas 148 right tubes $(51.75 \%)$ and 152 left tubes $(53.15 \%)$ were demonstrated completely. Loculated contrast spill was observed in ten right and seven left tubes. Free spillage of contrast was observed in 138 right and 145 left tubes. Bilateral free contrast spillage was observed in 128 cases. Tubal occlusion was seen in 24 right (8.39\%) and 26 (9.09\%) left cases. Distal occlusion was maximum (20 cases each) than isthmic and cornual obstruction in both the tubes. Bilateral tubal occlusion was seen in 28 cases. Hydrosalpinx was observed in 22 cases $(7.69 \%)$ of right and $28(9.79 \%)$ of left cases. There was no spillage of contrast material in majority of hydrosalpinx cases in both left and right occlusion (Table 4).

\section{DISCUSSION}

Hysterosalpingogram is widely used as one of the method in assessing the tubal pathology among cases of infertility. As it is a cheap, convenient and safe procedure majority of the cases are screened with HSG in low income countries and remains as a primary choice of imaging modality. In the present study conducted, majority of the cases of infertility were secondary (88.81\%) as compared with the findings in the study of Masoumi SZ et al who reported $78 \%$ of cases in their study. ${ }^{5}$ The most common age group in our study was $36-$ 40 years with mean age of $35.16 \pm 2.8$ years which is on par with the reports of Boivin $\mathrm{J}$ et al who reported the infertility overall in the age group of 30-40 with mean age of 37 years. ${ }^{6}$ In our study, majority of the cases were literate $(82 \%)$ which differs with the study findings of Raymont A which was done in Kenya with more cases of infertility associated with illiterates than literates as in our 
study. ${ }^{7}$ Majority of the cases in our study belongs to lower middle class (57\%) as per modified Kuppuswamys classification but our findings do not coincide with the reports globally and were found not significant. ${ }^{8}$ No statistical significance was associated history of consanguineous marriage with relation to infertility in our study. $88.81 \%$ of cases had regular menstrual cycles in our study and observed in both primary and secondary cases of infertility; however few studies reported more number of irregular cycles in cases of primary infertility than secondary infertility. Mean duration of primary and secondary infertility was $5.42 \pm 2.6$ years and $5.34 \pm 3.5$ years in the present study and were on par with the finding in the reports of Luciano A et al, but the duration of infertility is variable from place to place due to multiple factors involved which may be hormonal causes, age group included in the study etc. ${ }^{9}$

In the present study, $25.17 \%$ of cases revealed normal findings on HSG and $74.83 \%$ were abnormal. This high incidence can be explained that more cases in our study were of secondary infertility than primary. In our study, $81.25 \%$ of cases of primary infertility had normal findings which are contrary to the findings in the study of Esmaeilzadeh S et al, who reported $67 \%$ of abnormal findings on HSG in cases of primary infertility. ${ }^{10}$ Findings in the study of Aflatoonian A et al, observed more normal findings in secondary infertility than primary cases which may be due to difference in the age group involved and cultural traditions observed. ${ }^{11}$ Our study revealed more uterine cavity anomalies $69.72 \%$ with more number of cases in secondary infertility than primary. Findings of our study were little high than the reports of Shah SM et al, who reported 56\% in their study which may be the reasons mentioned previously. ${ }^{12}$ Only one case of unicornuate and one case of bicornuate uterus was identified in the entire study and was similar to the reports mentioned in various studies with less than $1 \%$ in any study reported. The incidence of uterine filling effects in the study was around $70.27 \%$ with most of them were smooth and associated with leiomyoma in 27 cases. $(64.29 \%)$ Findings of our study correlate with the findings of Mgbor SO et al who made similar findings in their study. ${ }^{13}$ A few contrary studies have also been identified but this may be due to higher frequency of uterine synechiae attributed to infections and curettage.

In our study with the tubal findings on $\mathrm{HSG}, 42$ right tubes $(14.69 \%)$ and 44 left tubes $(15.38 \%)$ were not visualized completely, whereas 148 right tubes $(51.75 \%)$ and 152 left tubes $(53.15 \%)$ were demonstrated completely. Most of the studies recorded about $33 \%$ to $47 \%$ of tubal abnormalities in their studies. ${ }^{14}$ Bilateral tubal occlusion were seen in 28 cases and hydrosalpinx in $7.69 \%$ of right and $9.79 \%$ of left cases which were similar to the results in the study of Bello TO et al. ${ }^{15}$ Tubal obstruction was most common in right than left fallopian tube in our study, this condition is variable in different studies. Few studies reported more blockages in right fallopian tube because of previous history of appendicectomy or surgical infections. Distal site of tube obstruction is more common than other sites like corneal or isthnmic obstruction and is more commonly due to development of infections. ${ }^{16}$ Pelvic infections cause adhesions commonly at the ampullary region of the fallopian tubes leading to the blockage of the tubes. The accumulation of secretions within these blocked fallopian tubes causes the tubes to dilate resulting in hydrosalpinx.

\section{CONCLUSION}

To conclude, women presenting with infertility should be evaluated for tubal pathology. The tubal pathologies like occlusion or hydrosalpinx may be due to infections or post surgical sequelae. HSG remains one of the diagnostic backbone in infertility even availability of new modalities. HSG should be performed more early as the cases of secondary infertility are more commonly observed. HSG also has the additional advantage of identification of congenital anomalies of the uterus.

Funding: No funding sources Conflict of interest: None declared

Ethical approval: The study was approved by the Institutional Ethics Committee

\section{REFERENCES}

1. Mascarenhas. National, regional and global trends in infertility prevalence since 1990: a systematic analysis of 277 health surveys. PLOS Med. 2012.

2. Abubakar AP, Yusuf TS. The profile of infertility in a teaching hospital in North West Nigeria. Sahel Med J. 2014;17:7-11.

3. Ganguly S, Unisa S. Trends of infertility and childlessness in India: findings from NFHS Data. Facts Views Vis Obgyn. 2010;2(2):131-8.

4. Phaylim C, Hasafa Z, Bhattacharya S, Maheshwari A. Should a hysterosalpingogram be a first-line investigation to diagnose female tubal subfertility in the modern subfertility workup? Hum Reprod 2011;26:967-71.

5. Masoumi SZ, Parsa P, Darvish N, Mokhtari S, Yavangi M, Roshanaei G. An epidemiologic survey on the causes of infertility in patients referred to infertility center in Fatemieh Hospital in Hamadan. Iran J Reprod Med. 2015;13(8):513-6.

6. Boivin J, Bunting L, Collins J, Nygren K. International estimates of infertility prevalence and treatment-seeking: potential need and demand for infertility medical care. Hum Reprod. 2007;22:150612.

7. Raymont A, Arronet GH, Arrata W. Review of 500 cases of infertility. J Fertil. 1998;(14):141-53.

8. Saleem SM. Modified Kuppuswamy socioeconomic scale updated for the year 2019. Indian J Forensic Community Med. 2019;6(1):1-3.

9. Luciano A, Lanzone A, Goverde A. Management of female infertility from hormonal causes. Int $\mathbf{J}$ Gynecol Obstet. 2013;123:S9-S17. 
10. Esmaeilzadeh S, Farsi M, Nazari T. The cause of infertility frequency in the patients referring to Babol township fatemeh zahra infertility center from May 1996 to May 1998. J Mazandaran Univ Med Sci. 2002;12:29-34.

11. Aflatoonian A, Seyedhassani S, Tabibnejad N. The epidemiological and etiological aspect of infertility inYazd province of Iran. Iran J Reprod Med. 2009;7:117-22.

12. Shah SM, Towobola OA, Masihleho M. Diagnosis of fallopian tube patency. East Afr Med J. 2005;82(9):457-62.

13. Mgbor SO. Pattern of hysterosalpingographic findings in gynaecological patients in Enugu. Nig Med J. 2006;47:14-6.

14. Al Subhi T, Al Jashnmi RN, Al Khaduri M, Gowri V. Prevalence of tubal obstruction in the hysterosalpingogram of women with primary and secondary infertility. J Reprod Infertil. 2013;14(4):214-6.

15. Bello TO. Tubal abnormalities on hysterosalpingography in primary and secondary infertility. West Afr J Med. 2006;25(2):130-3.

16. Swart P, Mol BW, van der Veen F, van Beurden M, Redekop WK, Bossuyt PM. The accuracy of hysterosalpingography in the diagnosis of tubal pathology: a meta-analysis. Fertil Steril. 1995;64(3):486-91.

Cite this article as: Reddy GS, Jyothi G.

Hysterosalpingogram in evaluation of primary and secondary infertility: a two year study at a tertiary care hospital of South India. Int J Reprod Contracept Obstet Gynecol 2019;8:2202-7. 